Research Article

Open Access

Oleg Hmilyar

\title{
Person's Behavior Control in Stochastic Conditions of Symbolic Danger
}

DOI 10.1515/sigtem-2016-0007

\begin{abstract}
A person's behaviour is controlled by five independent regulators - symbol, image, thought, sense and feeling. In this article, the author describes particularities of a person's behaviour control in simulated stochastic conditions of symbolic danger. An ability of a person to control his/her behaviour under symbolic danger could be measured by a device designed for researching person's behaviour under stochastic conditions. It was defined that symbolic danger could harmonise a person's behaviour and cause him/her to act more constructively. The results of a psychological experiment indicated that under stochastic conditions of symbolic danger, efficiency of solving psychomotor tasks depends on the person's ability to act under situation of uncertainty in space and time and alternative. Sensor-perceptual and symbolic and visual spheres that developed in a proper way allow to provide constructive control of person's behaviour under situations related to lack of time or psychological tension. Control of behaviour under stochastic conditions of symbolic danger significantly depends on the level of energetic potential of a person. It was defined that participants of the psychological experiment with a high level of energetic potential acted more effectively under symbolic danger compared to persons with low energetic potential. In case of complication of psychomotor tasks, energetic potential of a person, in combination with sensor and motor skills, process of thinking and imagination, consolidate his/her behaviour in stochastic conditions of symbolic danger.
\end{abstract}

Keywords: symbol, stochastic conditions, personality, behaviour control, energetic potential.

\section{Introduction}

Among the main directions and spheres of modern psychological science, one significant tendency is definitely highlighted, that is, learning of a person by his/her subject qualities, abilities, processes and self-development conditions. Subjective human existence is complex and multispectral and is supported by a mental system and could be realised in different forms. One of the most general and essential exposure of person's subjectivity is his/her voluntary conscious activity by which a wide range of relations between person and world of things, people and conditions of environment is realised. Herewith, control of conscious activity under stochastic conditions in simulated symbolic danger is the most general function of integrated mentality. In the process of behaviour control under stochastic conditions of symbolic danger, a unity of person's mentality could be indicated from different aspects of his/her abilities and skills.

By controlling behaviour under stochastic conditions of symbolic danger, a person, first of all, has to regulate, adjust and develop his/her actions in accordance with the defined rules and norms. As person's behaviour is a part of a wide system of symbolic regulation, its main functions are assessment, support, protection and reproduction of norms, rules, mechanisms and means necessary for subjects of regulation that provides cooperation (Rubinstein, 2000).

*Corresponding author: Oleg Hmilyar: Department of social sciences, National Defence University of Ukraine named after Ivan Chernyakhovskyi, Ukraine, E-mail: hmilar@ukr.net 
Regulation of person's behaviour under stochastic conditions of symbolic danger is a task of significant complexity as it predicts hormic usage of proper actions needed for achieving meaningful goals. The same actions, depending on prediction of outcome or product of action and sense of task, are guided and corrected minimum by five independent regulators: symbol, thought, image, sense and feelings (Maksimenko, Zaichuk and Klimenko, 2000).

\section{The Aim of the Study}

The aim of the article is to find out how stochastic conditions of symbolic danger influences a person's behavior control.

\section{Materials and Methods}

In order to simulate stochastic conditions that allow to observe particularities of influence of symbolic danger on person's ability to control his/her behaviour, a 'device for researching person's behaviour under stochastic conditions', designed and patented by us, was used in the experiment (Fig. 1). The device allows to harmonise motions of a person and monitor influence of his/her energetic potential on his/her actions (V.V.Klimenko \& O.F. Hmilyar, 2005).

Construction of the device is analogical to those that were used by warriors in Ancient Sparta. Its originality is that a person being researched acts under conditions that force him/her by making different motions (like bending body, squatting, jumping off) to overcome approaching obstacles such as rotating bars in horizontal space at different altitudes. The time and character of obstacle that define technique of action can be both known in advance and unknown to a person being researched.

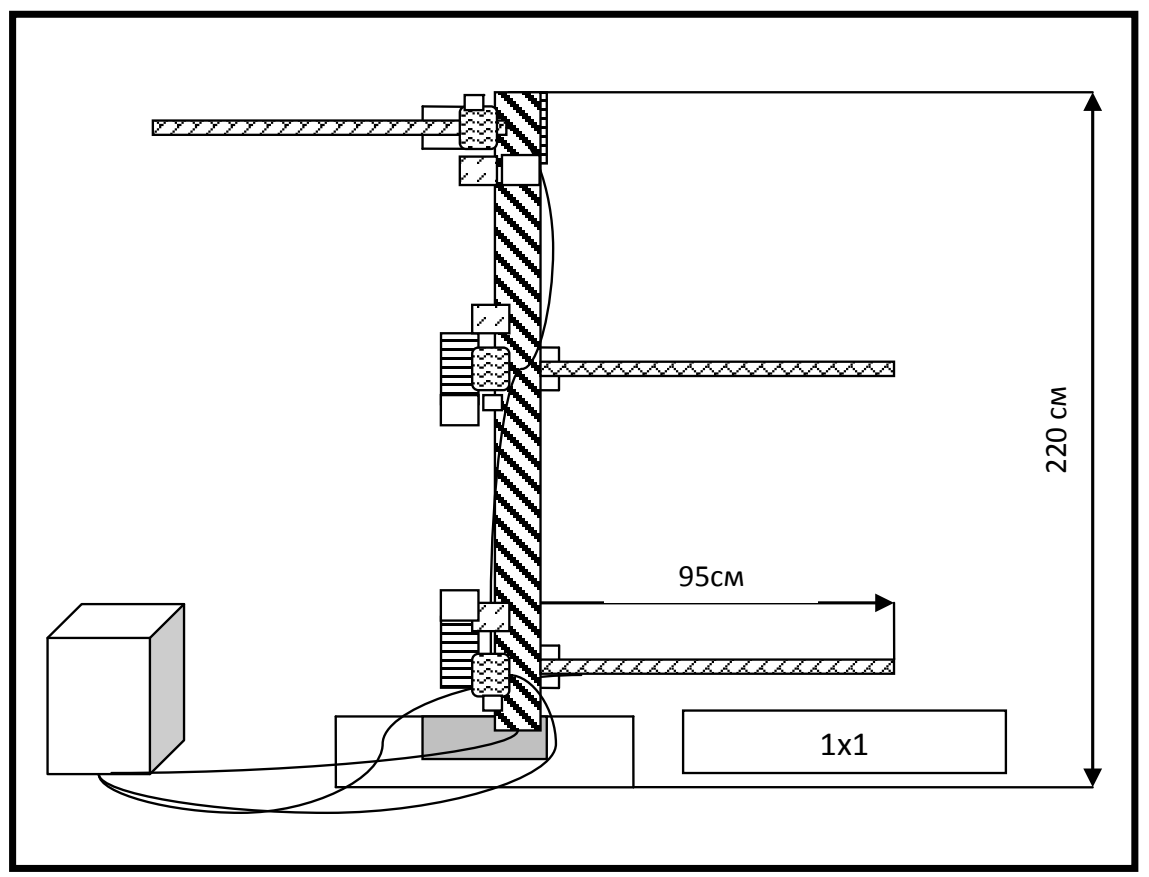

Fig. 1. Device for researching person's behaviour under stochastic conditions

A program (depending on a character of tasks includes 12-24 stimuli) is directed from a control panel. In accordance with the program, the device works in an automatic mode. 
Stochastic conditions of symbolic danger are created by changing direction of the rotation of by urgent acceleration or delay in the movement and by simultaneous input of two stimuli (obstacles). The height of obstacles depends on person's height: the first bar is installed at a level of person's ankle joint and it should be overcome by jumping it off, the second bar is installed at chest level and should be overcome in a deep squat, the third bar is installed at chin level and to overcome it, you should bend your head. The speed of rotation is one rotation per second. To perform one task, one should overcome obstacles for 6-18 times. One could make two to three attempts. The best result will be taken into account when less mistakes are made related to touching and delaying rotation of a bar. The time of touching a bar by a person being researched is registered in milliseconds by an automatic device.

In the psychological experiment, bars approached in different time space with sudden acceleration and delay of rotation, simultaneous appearance of two obstacles, different speeds and sequence of rotations. A combination of these elements allowed to create symbolic danger that should have resulted in significant influence on actions of persons being researched.

Officers of the Armed Forces of Ukraine under 29-33 years were the subjects in this study ( $n=89$, male). All participants of the psychological experiment were healthy and systematically mastered their psychomotor abilities. Before the experiment took place, persons being researched measured their energetic potential (Klimenko, 2000). Based on the obtained results, two groups were formed: first group consisted of persons with high energetic potential ( $n=42$ persons), the second group consisted of persons with low energetic potential ( $n=47$ persons).

Particularity of symbolic danger was that one of persons being researched under simulated stochastic situations acted in more favourable conditions. All his peers cheered him up to help to make as less mistakes as possible. Another person being researched (his 'opponent') had to overcome approaching obstacles under least favourable conditions (at that moment all his peers were in another room). A person being researched definitely understood that he was 'captured' by the adversary group. Therefore he had to be much more attentive and focused on his actions. In general, the psychological experiment in stochastic symbolic danger conditions was simulated in a way that each participant was able to control his behaviour under most favourable and least favourable conditions.

\section{Results}

Interpretation of obtained results in the psychological experiment indicated significant difference in actions of persons with high and low levels of energetic potential (see Table 1). Table 1 indicates that the worst results showed persons with low energetic potential who acted in conditions of symbolic danger: 'I am one to one with adversary', 'I act in adversary group', in other words, who were under least favourable conditions. These results were confirmed by average mistakes measured in time of touching an obstacle: in the first case its average was 15.22" $(P<0.05)$, in the second case -18.53 ” $(P<0.05)$.

Table 1. Dynamics of influence of symbolic danger on behaviour control under stochastic conditions by persons being researched

\begin{tabular}{|c|c|c|c|c|c|c|c|c|}
\hline \multirow{2}{*}{$\begin{array}{l}\text { Degree of } \\
\text { complexity }\end{array}$} & \multirow{2}{*}{$\begin{array}{l}\text { Simulated } \\
\text { conditions }\end{array}$} & \multirow{2}{*}{ Symbolic danger } & \multirow{2}{*}{ Groups } & \multirow{2}{*}{$\begin{array}{l}\text { Average } \\
\text { mistake (sec) }\end{array}$} & \multicolumn{4}{|c|}{ Another statistic data } \\
\hline & & & & & $\sigma$ & Thickest value & Median & CV \\
\hline \multirow{8}{*}{$\begin{array}{l}\text { Actions of } \\
\text { higher com- } \\
\text { plexity }\end{array}$} & \multirow{8}{*}{$\begin{array}{l}\text { Stochastic } \\
\text { conditions }\end{array}$} & 'I am one to one with & 1 & $12.17^{\star}$ & 0.22 & 12.24 & 12.20 & 1.80 \\
\hline & & adversary' & 2 & 15.22 & 1.03 & 14.27 & 15.05 & 6.76 \\
\hline & & 'I am in an adversary & 1 & 10.44 & 1.13 & 10.57 & 10.51 & 10.82 \\
\hline & & group' & 2 & 18.53 & 2.26 & 19.07 & 18.98 & 12.19 \\
\hline & & 'I am in a friendly & 1 & $9.98^{\star}$ & 0.74 & 10.06 & 10.02 & 7.41 \\
\hline & & group' & 2 & $14.87^{\star}$ & 2.08 & 14.35 & 14.73 & 13.08 \\
\hline & & Check measurement & 1 & $10.02^{\star}$ & 1.04 & 10.23 & 10.46 & 10.37 \\
\hline & & & 2 & 15.06 & 1.82 & 15.31 & 15.64 & 12.08 \\
\hline
\end{tabular}

References: 1 - persons with high energetic potential $(n=42) .2$ - persons with low energetic potential $(n=47)$.

* - probability of difference in group when $P<0.05$. 
The highest results persons in the second group demonstrated under conditions 'I act in a friendly group'. The average mistake in this situation was 14.87" $(P<0.05)$. In a check measurement in a situation when each officer acted for himself, only average mistake was 15.06" $(P<0.05)$.

The average mistake of persons being researched with high energetic potential under uncomfortable conditions 'I am one to one with adversary' was 12.17" $(P<0.05)$ and 10.44" under 'I act in an adversary group'. The highest results in the first group were demonstrated under situation 'I act in a friendly group' (average mistake was 9.98").

Therefore, the results of research obtained under simulated stochastic conditions indicated that symbolic danger could reduce person's ability to control his/her behaviour and at the same time cause more constructive actions. This statement confirmed hypothesis made by M. O. Bernstein on 'rationality law', which states that in the case of highest pressure when nerves and muscles are 'strained to the limit', a body cannot move and act for waste. Therefore actions are done properly in the best way (Bernstein, 1966).

Symbolic danger, in connection with high energetic potential, caused persons in the first group to move and act in a maximal rational way. Therefore, they achieved results that were not possible to achieve under normal circumstances.

The results of the psychological experiment also confirm S. G. Gellerstein's idea that particularity of any psychomotor task is significantly defined by sensor-perceptual and symbolic and image activities that determine condition for decision-making process (Gellerstein, 1966).

In solving of psychomotor tasks in previously known conditions, efficiency of actions is largely defined by requirements to motor abilities (in our case, speed of moves), while in stochastic conditions of symbolic danger effective control of behaviour is limited mainly by identical requirements to mental processes and qualities.

\section{Conclusions}

The results of researching person's behaviour control in stochastic conditions of symbolic danger allowed to indicate a close relation between mental and motor components in solving motional tasks. In support of Bernstein's idea that efficiency of behaviour control in stochastic conditions is significantly dependent on extraversion, the ability to improvise and inventiveness, we came to a conclusion that among mental and motor abilities, the priority is given to mental ability. Mental qualities (such as quick perception of a situation and its assessment, ability to quickly select a proper motion - quick decision-making), ability to create state of 'public loneliness' are effective regulators of person's behaviour who acts under stochastic conditions of symbolic danger.

By complicating conditions under which participants had to make decisions, a value of technique of psychomotor activity was reduced as the final outcome depended less on motion. Instead, a significant role demonstrated nerve and muscles system, sensor-motor skills, process of thinking, and feeling and imagination.

\section{References}

Bernstein, N. A. (1966). The Sketches on Motion Physiology and Activity Physiology. Moscow: Medicine.

Gellerstein, S. G. (1966). Activities Based on Anticipation and Possibility to Simulate them in Experiment. Problems of Engineering Psychology. Ed. 4. p. 34-39.

Klimenko, V. V., Hmilyar, O. F. (2005). Device for Researching Person's Behavior in Stochastic Conditions. Utility model declaration patent. - Ukraine, State Department on Intellectual Property. (19) UA (11) 7956 (13) U (51) 7 A63B24/00. Bul. \# 7,6 p.

Maksimenko, S. D., Zaichuk, V. O., Klimenko, V. V. (2000). General Psychology. Kyiv: Forum.

Rubinstein, S. L. (2000). Basics of General Psychology. St. Petersburg: Piter. (in Russian) 